\title{
A smeared-embedded mesh-corrected damage model for tensile cracking
}

\author{
M. Cervera*, $\dagger$ \\ International Center for Numerical Methods in Engineering (CIMNE), Technical University of Catalonia (UPC), \\ Edificio C1, Campus Norte, Jordi Girona 1-3, 08034 Barcelona, Spain
}

\begin{abstract}
SUMMARY
Traditional smeared orthotropic models display an unacceptable dependence of the solution on the alignment of the mesh, which usually manifests as stress locking. A solution for this drawback is proposed in this paper by adopting the concept of embedded inelastic strains, rather than displacement jumps, and by linking the structure of the inelastic strain to the geometry of the cracked element. The resulting model, applicable to linear 3-noded triangles, is formulated as a non-symmetric orthotropic local damage constitutive model, with the softening modulus regularized according to the material fracture energy and the element size. Analytical and numerical results show that this approach is effective in removing the locking problem as well as efficient from the computational point of view. Copyright (C) 2008 John Wiley \& Sons, Ltd.
\end{abstract}

Received 12 April 2007; Revised 25 March 2008; Accepted 23 April 2008

KEY WORDS: tensile cracking; orthotropic damage; strain softening; shear locking; smeared-embedded models

\section{INTRODUCTION}

The modelling of tensile cracks has been a subject of earnest interest, both professional and academic, since the earliest applications of the finite element (FE) method to concrete structures, back in the 1960s. For more than 40 years, many different strategies for crack modellization have been suggested and new terminology has been coined.

From the very beginning [1,2], two alternative approaches have been used: the discontinuous and the continuous crack approaches. In the discontinuous (also known as 'discrete') crack models, the displacement field is discontinuous across the crack and the cohesive behaviour of this is

\footnotetext{
${ }^{*}$ Correspondence to: M. Cervera, International Center for Numerical Methods in Engineering (CIMNE), Technical University of Catalonia (UPC), Edificio C1, Campus Norte, Jordi Girona 1-3, 08034 Barcelona, Spain.

†E-mail: miguel.cervera@upc.edu

Contract/grant sponsor: SEDUREC; contract/grant number: CSD2006-00060
} 
established through a softening traction-separation law. Contrariwise, in the continuous (also known as 'smeared') crack models, displacement jumps across the crack are smeared over the affected elements and the overall behaviour is established through a softening stress-(total) strain law.

The first option is mostly used in the context of Fracture Mechanics, whereas the second is frequently adopted in Continuum Mechanics. In addition, the smeared crack concept is more easily implemented in a standard non-linear FE code, and this is the reason why most structural engineers favour this approach. However, the observed mesh size and mesh bias dependence exhibited by these models have made the academic world very suspicious about this format. In fact, a lot of effort has been spent in the last decades to explain the observed drawbacks of this approach.

However, the most promising of the newly proposed methods resign from the smeared approach and turn back to the discontinuous format. Here, we can mention the extended finite element method (X-FEM) [3-5], which fits in the Fracture Mechanics framework, and the so-called strong discontinuity approach [6-16], which, starting from Continuum Mechanics, leads to enhanced formulations for FEs with embedded displacement discontinuities.

Nevertheless, the experience gained from the new embedded formulations opens the possibility of a novel version of smeared models, here termed as smeared-embedded crack models, which incorporate the effect of the displacement jumps in the strain field of the elements, rather than the actual jumps themselves. In such models, on one hand, all computations are made at a constitutive level and this allows the implementation they introduce the $n$

Accordingly, the locking problems ass conceptual comparis on
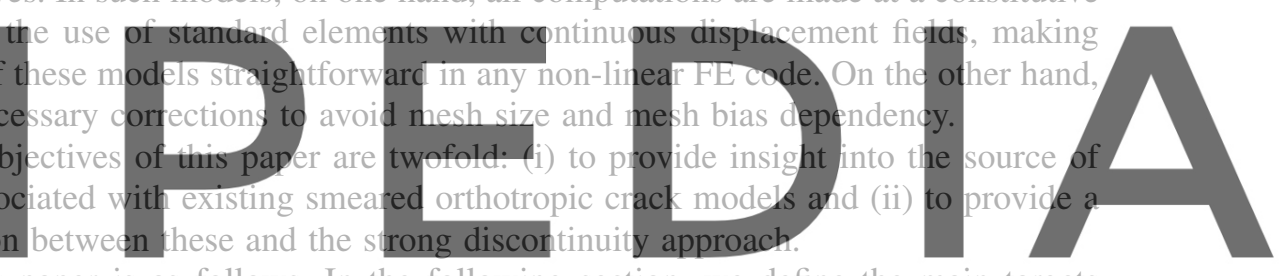

The outline of this paper is as follows. In the following section, we define the main targets

is presented. It is shown that this model successfully overcomes the difficulties presented by the classical orthotropic smeared models. Finally, selected numerical examples are presented to assess the proposed formulation.

\section{THE SMEARED-EMBEDDED CRACK APPROACH}

In this section, we introduce the smeared-embedded approach, a variation of the original smeared approach [2], with inputs from the so-called embedded discontinuities or strong discontinuity approach [6]. We will consider both the formulation at continuum level and its discrete counterpart, the FE implementation.

The FE implementation is restricted to 2D meshes with linear 3-noded FE. Even if the limited approximation capabilities of this CST element are well known, it is vastly used in practical applications due to its simplicity and easiness of use. Two considerations can be made related to this. On one hand, extension of the present work to quadrilateral elements in 2D may proceed along the ideas presented in References $[13,14]$. As it is shown in the numerical applications shown there, the benefits of using quadrilaterals over triangles are evident in coarse meshes; however, crack propagation problems always require fine meshes along the crack path and, thus, those 
benefits do not show so patently. On the other hand, the extension of the present approach to 3D meshes with linear 4-noded tetrahedra seems relatively simple.

\subsection{Continuum setting}

Consider an elastic body $\Omega$, shown in Figure 1(a), crossed by a discontinuity $S$, which represents a crack. Regions $\Omega^{+}$and $\Omega^{-}$are the parts of the body located 'in front' and 'behind' the crack.

The smeared continuum crack model is represented in Figures 1(a) and 2(a). Here, $S^{+}$and $S^{-}$ are two lines that run parallel to $S$, at a relative distance of $h$, measured in the direction normal to the crack. In this model, the normal jump $w$ occurring at $S$ is smeared over the distance $h$. The top graph in Figure 2(a) shows the normal displacement along a line normal to the crack, with the normal jump $w$ smeared continuously between $S^{-}$and $S^{+}$. The corresponding normal strain is shown in the bottom graph, with no discontinuity occurring at $S$. The adoption of a continuous displacement description for the crack allows to formulate the constitutive behaviour in terms of a softening stress-(total) strain law, thus avoiding the need of establishing an ad hoc traction-jump cohesive law.

The smeared-embedded continuum crack model, represented in Figure 2(b), is introduced as a refinement of the basic smeared continuum model. In it the displacement field is continuous inside the localization band but, as in other embedded models, the strain field is decomposed into its
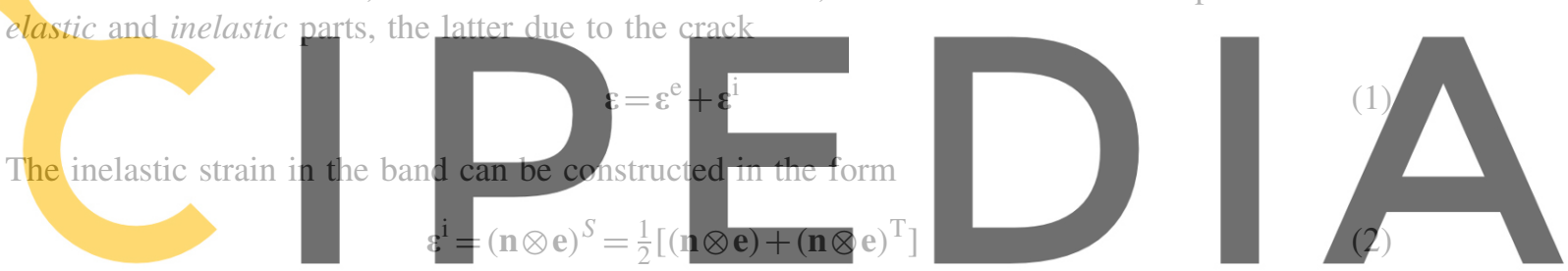

where $\mathrm{n}$ is a unit vector normal to the crack and $\mathrm{e}$ is the inelastic deformation vector on the plane

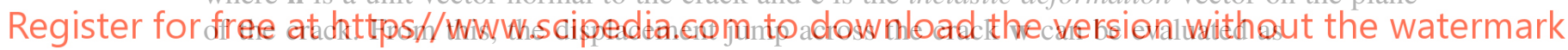

$$
\mathrm{W}=h \mathrm{e}
$$

Once the inelastic part of the strain is discounted, the stress can be related to the remaining elastic part. The inelastic deformation vector $\mathbf{e}$ and, therefore, the contribution of the crack to

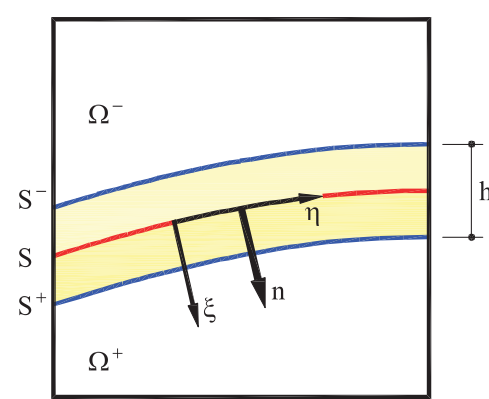

(a)

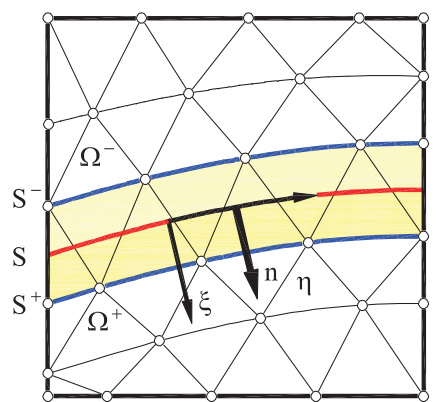

(b)

Figure 1. Modellization of a crack by the smeared approach: (a) continuum level and (b) discrete level. 


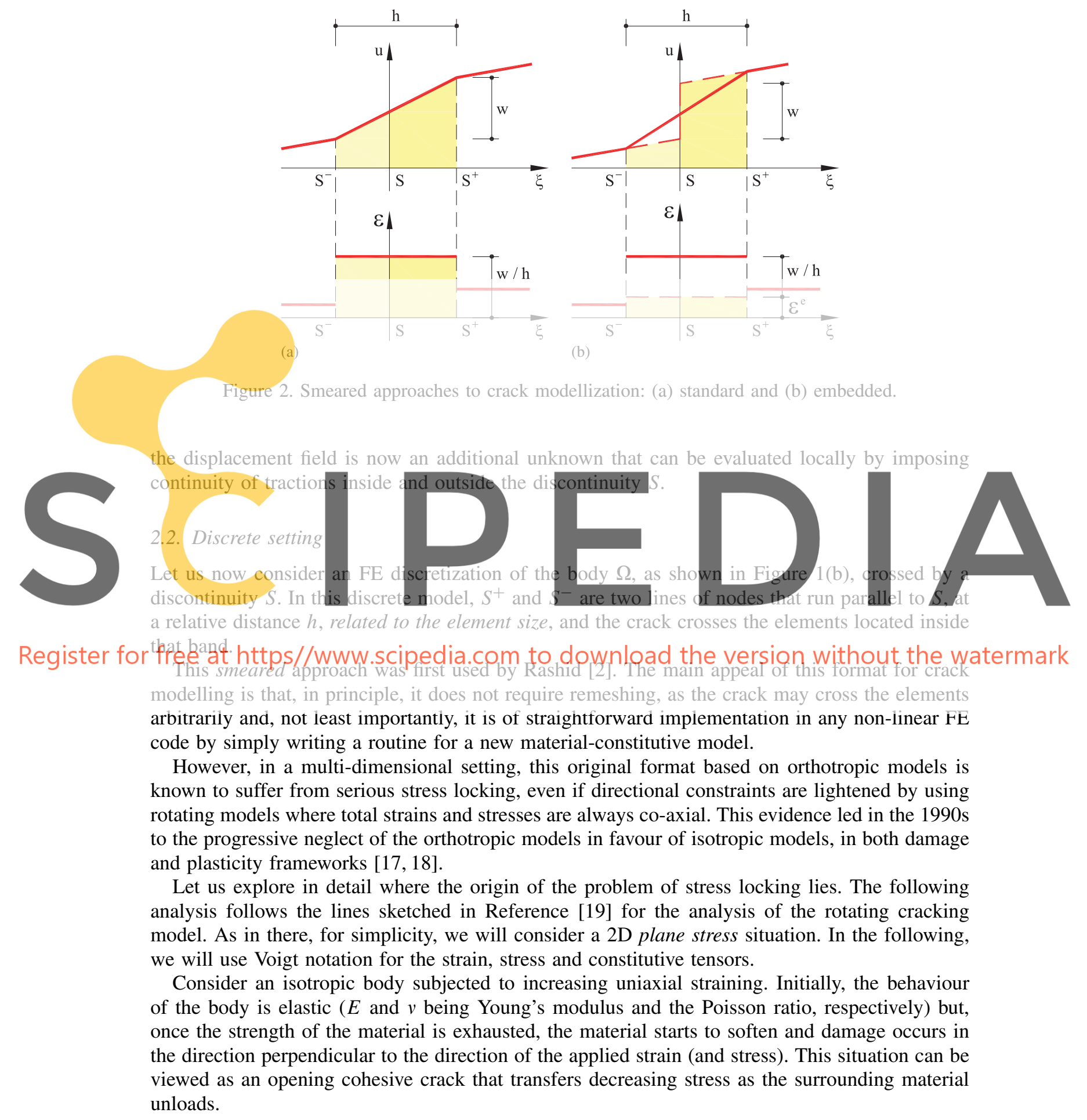


Let us now consider the discrete model depicted in Figure 3, consisting of a linear 3-noded triangle crossed by a line discontinuity representing a crack. Global coordinate axes are introduced, such that the $x$-axis is aligned with the applied straining and the crack and the $y$-axis are orthogonal to them. The strain field inside the CST can be expressed, in matrix form, as

$$
\left[\begin{array}{c}
\varepsilon_{x} \\
\varepsilon_{y} \\
\gamma_{x y}
\end{array}\right]=\frac{1}{2 A}\left[\begin{array}{cccc}
y_{3}-y_{1} & 0 & y_{1}-y_{2} & 0 \\
0 & x_{1}-x_{3} & 0 & x_{2}-x_{1} \\
x_{1}-x_{3} & y_{3}-y_{1} & x_{2}-x_{1} & y_{1}-y_{2}
\end{array}\right]\left[\begin{array}{c}
u_{2}-u_{1} \\
v_{2}-v_{1} \\
u_{3}-u_{1} \\
v_{3}-v_{1}
\end{array}\right]
$$

where $A$ is the area of the element, $x_{i}$ and $y_{i}$ are the nodal coordinates and $u_{i}$ and $v_{i}$ are the horizontal and vertical components of the nodal displacements, respectively.

Before cracking occurs, the displacement differences can be expressed as

$$
u_{i}-u_{j}=\overline{\varepsilon_{x}}\left(x_{i}-x_{j}\right), \quad v_{i}-v_{j}=-v \overline{\varepsilon_{x}}\left(y_{i}-y_{j}\right)
$$

where $\overline{\varepsilon_{x}}$ represents the homogeneous strain applied in the $x$-direction. As expected, using expressions (5) in (4) yields the exact strain field:
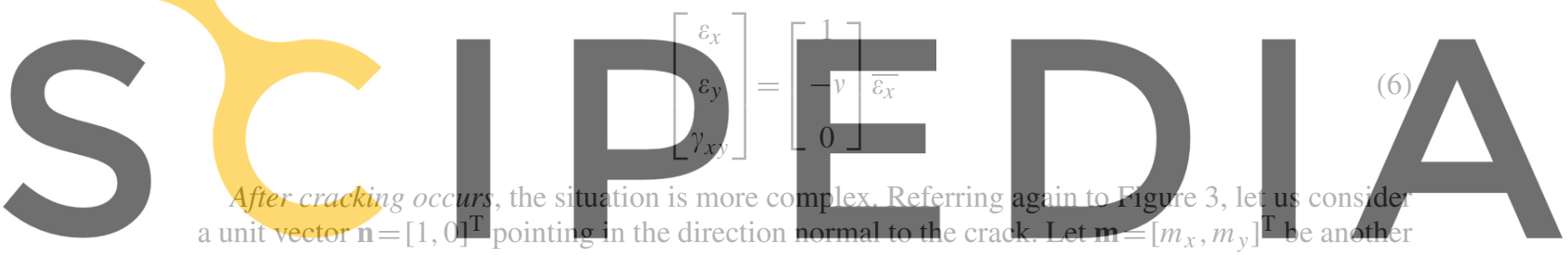

unit vector that is selected as the normal to the element side that maximizes the value of the

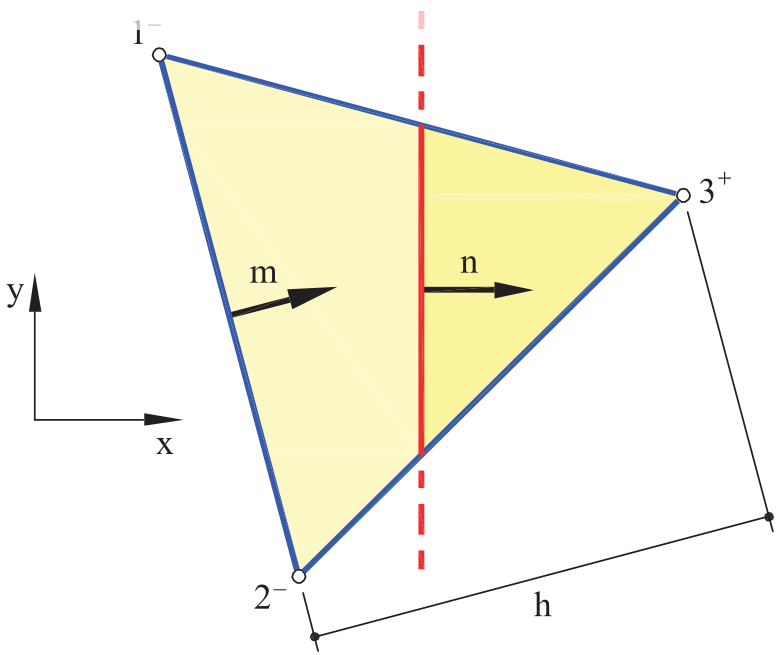

Figure 3. Three-noded plane stress triangle subjected to uniaxial straining. 
We will assume that the crack does not intersect the side $1^{-}-2^{-}$and that it separates this side from node $3^{+}$(solitary node). Vector $\mathbf{n}$ is directed so that it points to $3^{+}$and vector $\mathbf{m}$ is directed so that the product $\mathbf{n} \cdot \mathbf{m}>0$.

In addition, we will take the width of the localization band, $h$, as the distance from the solitary node $3^{+}$to the opposite side $1^{-}-2^{-}$. This allows one to identify the contribution of the crack to the displacement field inside the element as $\mathbf{w}=h \mathbf{e}$. Note that this 'displacement jump' need not be co-axial with vector $\mathbf{n}$ or with vector $\mathbf{m}$. The appropriate selection of length $h$ is very important in the FE implementation of a smeared model, as it affects the value of the discrete softening parameter, as it will be shown below. In an orthotropic model, where damage is associated with a specific damage plane, $h$ must be selected according to the orientation of this plane. Contrariwise, in an isotropic model, where damage does not have a directional character, it is usual to select length $h$ related to the element diameter.

After cracking, the applied straining $\overline{\varepsilon_{x}}$ can be decomposed into an elastic part $\left(\overline{\varepsilon_{x}}\right)^{\mathrm{e}}$ and an inelastic part that we can express as $\left(\overline{\varepsilon_{x}}\right)^{\mathrm{i}}=\overline{w_{x}} /\left(x_{3}-x_{1}\right)$, where $\overline{w_{x}}$ is the opening of the crack and $x_{3}-x_{1}$ is the projection of the element in the direction orthogonal to the crack.

As nodes $1^{-}$and $2^{-}$are located on the same side of the crack, the difference $u_{2}-u_{1}$ does not vary from the pre-crack situation. However, difference $u_{3}-u_{1}$ is now

$$
u_{3}-u_{1}=\left[\left(\overline{\varepsilon_{x}}\right)^{\mathrm{e}}+\left(\overline{\varepsilon_{x}}\right)^{\mathrm{i}}\right]\left(x_{3}-x_{1}\right)
$$
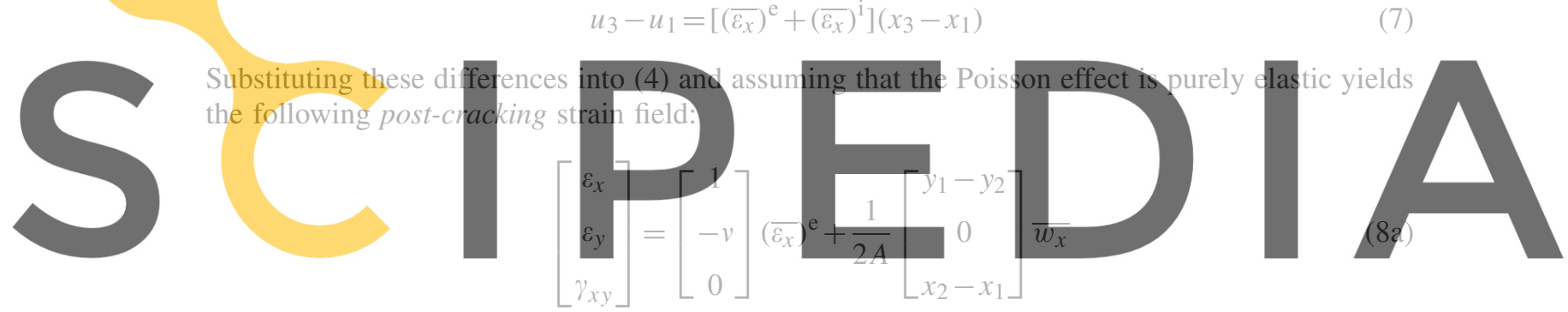

Register for free at https//www.scipedia

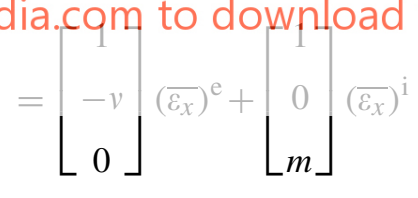

(8b)

where $m=m_{y} / m_{x}=\left(y_{1}-y_{2}\right)\left(x_{3}-x_{1}\right) / 2 A$ is the tangent of the angle between vectors $\mathbf{m}$ and $\mathbf{n}$. Note that the inelastic components of the strain are proportional to the opening of the crack $\overline{w_{x}}$, but the structure of the inelastic strain vector depends on the direction of vector $\mathbf{m}$, that is, on the orientation of side $1^{-}-2^{-}$with respect to the crack, a feature specific to the spatial discretization used in the modellization.

Let us introduce a scalar $\beta=\left(\overline{\varepsilon_{x}}\right)^{\mathrm{i}} /\left(\overline{\varepsilon_{x}}\right)$ as the ratio of the inelastic to the total strain in the $x$-direction. We can rewrite Equation $(8 b)$ as

$$
\boldsymbol{\varepsilon}=\left[\begin{array}{c}
\varepsilon_{x} \\
\varepsilon_{y} \\
\gamma_{x y}
\end{array}\right]=\left[\begin{array}{c}
1 \\
-v(1-\beta) \\
m \beta
\end{array}\right]\left(\overline{\varepsilon_{x}}\right)
$$

Figure 4 (left) represents the evolution of the three strain components as the axial strain increases, for a case with $\alpha=\arctan m=-30^{\circ}, m=-\sqrt{3} / 3$. The assumed material properties are Young's 

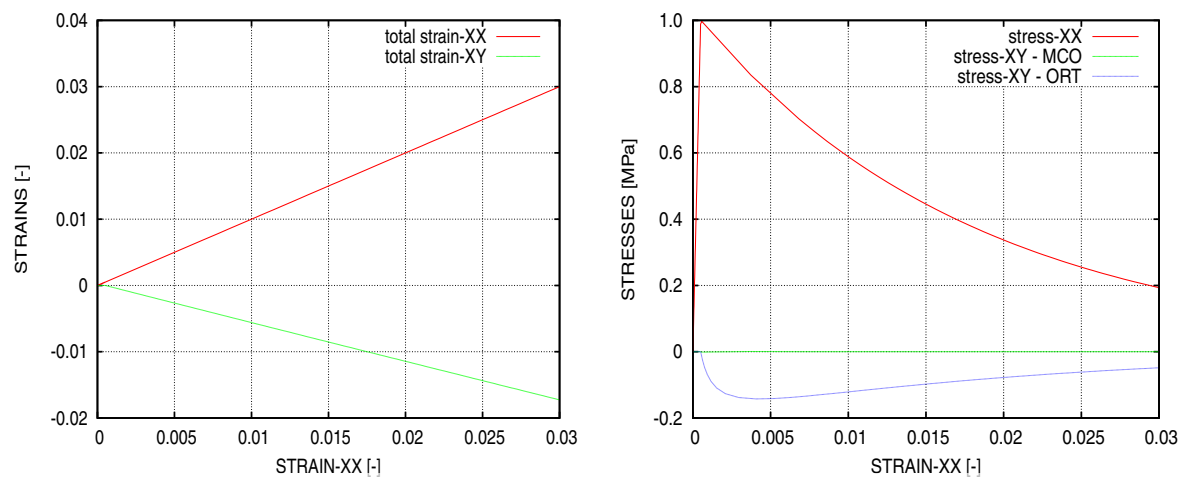

Figure 4. Strains and stresses developed in the discrete model.

modulus $E=2 \mathrm{GPa}$, the Poisson ratio $v=0.3$, tensile strength $\sigma_{0}=1 \mathrm{MPa}$ and mode I fracture energy $G_{\mathrm{f}}=250 \mathrm{~J} / \mathrm{m}^{2}$. Note how the spurious shear strain is directly proportional to the inelastic axial strain. This 'inelastic' shear strain component depends in an obvious manner on the orientation of the mesh and it only vanishes if vector $\mathbf{m}$ coincides with vector $\mathbf{m}$, that is, if the crack is parallel to one of the sides of the FF, $(m=0)$. This phenomenon appears due to the limitations of the spatial discretization; model. Therefore, the properly the inelastic $\left(\overline{\gamma_{x y}}\right)^{i}=m \beta\left(\overline{\varepsilon_{x}}\right)$.

This sort of limitation due to the spatial discretization is not ex fracture. A similar difficulty appears when using incompressible von Mises-type softening models. Also in that case the discrete problem has to be modified appropriately to obtain mesh-independent Register for fereres atohttps//www.scipedia.com to download the version without the watermark

Inspection of the total strain vector in Equation (9) shows that to obtain the correct elastic stress vector, the inelastic material model should be able to provide inelastic strains with the structure

$$
\boldsymbol{\varepsilon}^{\mathrm{i}}=\left[\begin{array}{c}
1 \\
0 \\
m
\end{array}\right]\left(\beta \overline{\varepsilon_{x}}\right)
$$

which can be expressed as

$$
\boldsymbol{\varepsilon}^{\mathrm{i}}=\mathbf{M}_{\mathrm{e}} \mathbf{e}=\left[\begin{array}{cc}
m_{x} & 0 \\
0 & m_{y} \\
m_{y} & m_{x}
\end{array}\right]\left[\begin{array}{c}
\beta \overline{\varepsilon_{x}} / m_{x} \\
0
\end{array}\right]
$$

where $\mathbf{e}$ is the inelastic deformation vector on the plane of the crack. Note that $e_{x}=\beta \overline{\varepsilon_{x}} / m_{x}=\overline{w_{x}} / h$.

Matrix $\mathbf{M}_{\mathrm{e}}$, constructed from the components of vector $\mathbf{m}$, is the Voigt representation of the projection tensor $\left(M_{\mathrm{e}}\right)_{i j k}=\frac{1}{2}\left(\delta_{j k} m_{i}+\delta_{i k} m_{j}\right)$. In fact, we can write that matrix $\mathbf{M}_{\mathrm{e}}$ is simply

$$
\mathbf{M}_{\mathrm{e}}=h \mathbf{B}_{3^{+}}
$$


where $\mathbf{B}_{3^{+}}$is the displacement-strain sub-matrix corresponding to the solitary node $3^{+}$. Therefore, the inelastic strain matrix $\boldsymbol{\varepsilon}^{\mathrm{i}}$ can be written as

$$
\varepsilon^{\mathrm{i}}=\mathbf{M}_{\mathrm{e}} \mathbf{e}=\mathbf{B}_{3}+\mathbf{w}
$$

where $\mathbf{w}=h \mathbf{e}$ represents the displacement jump at the crack. On one hand, this clarifies the smeared nature of this model and, on the other hand, shows its close relation with the discontinuousembedded models and with the enhanced assumed strain methods.

\section{ORTHOTROPIC RANKINE DAMAGE MODEL}

Classical smeared crack models are formulated as hyperelastic constitutive models, based on stress vs total strain relationships. In them, the material is initially isotropic, and a Rankine criterion, based on maximum tensile stress or strain, is used to define the onset of cracking. Thereafter, the behaviour is considered orthotropic, with different stiffnesses in the directions orthogonal and parallel to the crack. The Poisson effect is usually neglected after cracking.

However, it was soon observed that, in the discrete problem, orthotropic models lead to locking caused by spurious shear strains. Rotating crack models, in which the axes of induced orthotropy

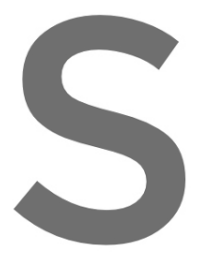
rotate as the principa adoption of these coit does not correct th because the FEs that strains and stresses as is spoiled by these strains do, were proposed to
rotational models avoids the
e basic difficulty in locking
implement the model are stan
the fixed models [19]. Furthe
purious contributions. to alleviate this pr
he question of she
g due to spurious
standard, and they
rthermore, the reoblem. Unfortunately, ev
ar transfer on the crack
shear inelastic straining
develop the same spurio
rientation of the orthotr even if the
ked plane,
ng. This is
rious shear

After 1990, it was observed that isotropic damage models mitigate to a large extent the problem

problem that affects the classical crack models. In fact, it is easy to show that the framework of continuum damage mechanics is very similar to the format of the original smeared crack models [29]. In this paper, we recover the tradition of those orthotropic models, reformulated within the CDM theory, and introducing a corrective mesh-dependent term to overcome the shear locking difficulty.

In this section we define an orthotropic damage model that depends on the definition of two physical directions aligned with two unit vectors $\mathbf{n}$ and $\mathbf{m}$. As introduced in Section 2.2 and shown in Figure 3, vector $\mathbf{n}$ is orthogonal to the damaged plane and vector $\mathbf{m}$ determines the structure of the inelastic strains (or stresses). For a classical Rankine model, damage occurs in a plane orthogonal to the major principal strain/stress and, therefore, this is the direction of vector $\mathbf{n}$. It is also standard, at continuum level, to consider that $\mathbf{m}=\mathbf{n}$. However, in the discrete problem, when the behaviour of FEs is considered, it is convenient to consider two separate vectors, $\mathbf{m}=\mathbf{n}+\boldsymbol{\delta}$, where $\boldsymbol{\delta}$ is a mesh-dependent correction to $\mathbf{n}$.

We have chosen a stress vs total strain format for the secant constitutive relationship because this fits well with the tradition in damage mechanics and smeared crack models. However, it is also possible to establish a rate tangent constitutive relationship. This would be the natural choice for a plasticity-type model. 
Finally, a comment is necessary about the alternative between fixed and rotating smearedembedded models. Obviously, both are defined depending on whether the directions identified by vectors $\mathbf{n}$ and $\mathbf{m}$ are kept fixed after the onset of cracking or whether they are updated thereafter according to the principal directions of strain/stress. Practical applications show that fixed models only work satisfactorily if the direction of straining does not change significantly during the loading process; otherwise, the locking difficulties progressively reappear. The first case occurs when cracks form completely at a given time and do not propagate; the second, much more frequent case, is typical of cracks that propagate with time across the domain of analysis. It is very possible that a model established in terms of a rate tangent constitutive relationship would circumvent this problem.

\subsection{Inelastic deformation and traction}

Let us begin by introducing the inelastic deformation vector e. In the smeared crack model, the inelastic deformation vector e can be viewed as the smearing, over a length $h$, of the displacement jump w across the crack, so that $\mathbf{w}=h \mathbf{e}$. This vector is associated with a physical direction in space identified by a unit vector $\mathbf{n}$ and the plane orthogonal to it (the plane of the 'crack'). The traction vector acting on this plane is

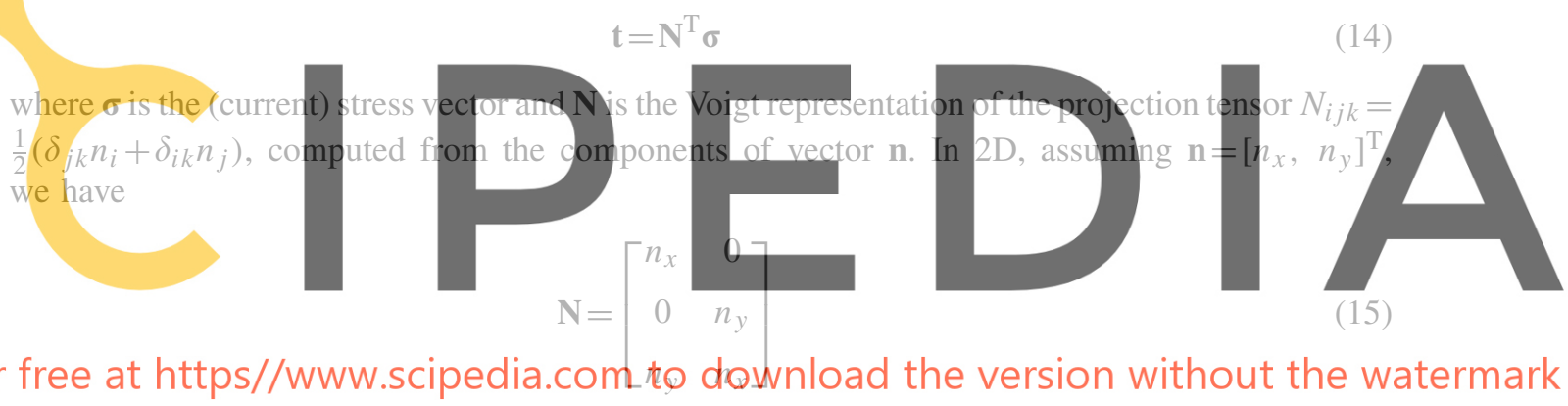

Note that matrix $\mathbf{N}$ has the same structure as the matrix $\mathbf{M}_{\mathrm{e}}$ in Equation (12).

The constitutive behaviour on the crack plane is determined by the traction vs inelastic deformation, $\mathbf{t}$ vs e, relationship. Formally, we can express

$$
\mathbf{t}=\widehat{\mathbf{H}} \mathbf{e}=\widehat{\boldsymbol{\Gamma}}^{-1} \widehat{\mathbf{C}} \mathbf{e}
$$

where $\widehat{\boldsymbol{\Gamma}}$ is a symmetric traction compliance matrix and $\widehat{\mathbf{C}}$ is a symmetric reference stiffness matrix.

Working in local coordinates for which the first axis is aligned with vector $\mathbf{n}$, symmetry arguments prove that the reference stiffness matrix must be diagonal. A natural option is to take $\widehat{\mathbf{C}}=\mathbf{N}^{\mathrm{T}} \mathbf{C N}$, where $\mathbf{C}$ is the isotropic linear-elastic constitutive matrix of the undamaged material. This relates the reference stiffness of the crack to the elastic moduli of the material, without the need of introducing additional parameters in the model.

As the traction compliance matrix is defined as a function of the traction, $\widehat{\Gamma}=\widehat{\Gamma}(\mathbf{t})$ or, alternatively, of the inelastic deformation vector, $\widehat{\boldsymbol{\Gamma}}=\widehat{\boldsymbol{\Gamma}}(\mathbf{e})$, Equation (16) is non-linear.

For later use, let us also introduce the inelastic traction vector $\mathbf{s}$ acting on the crack plane in the form

$$
\mathbf{s}=\widehat{\mathbf{C}} \mathbf{e}
$$


so that Equation (16) can be expressed simply as

$$
\mathbf{t}=\widehat{\boldsymbol{\Gamma}}^{-1} \mathbf{s}
$$

\subsection{Traction compliance and damage}

Equations (16) and (18) depend on the definition of the traction compliance matrix $\widehat{\boldsymbol{\Gamma}}$, which defines the relationship between the inelastic deformation and the traction acting on the plane orthogonal to vector $\mathbf{n}$. Working in an orthonormal basis in which the first direction coincides with $\mathbf{n}$, the simplest compliance matrix takes the diagonal form

$$
\widehat{\boldsymbol{\Gamma}}=\frac{d}{1-d} \mathbf{I}
$$

where $d$, the damage index, is a scalar internal-like variable whose definition and evolution is discussed below. In the context of a damage model, it is natural to define this relation in terms of damage rather than compliance. The traction compliance matrix can be related to a corresponding traction damage matrix defined as

$$
\widehat{\mathbb{D}}=\left[\mathbb{I}+\widehat{\Gamma}^{-1}\right]^{-1}=d \mathbb{I}
$$

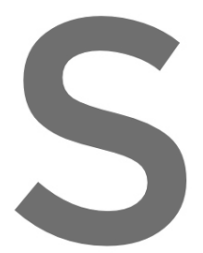

This simple definitio degrade in the same inate with different damag

3.3. The damage index

To complete the formulation of the damage model at the crack plane, it is necessary to define the evolution of the damage index, $d$, in terms of the eyolution of the effective stresses.or, equivalently,

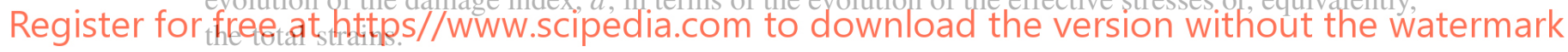

The Rankine-type damage criterion, $\Phi$, is introduced as

$$
\Phi(\tau, q)=\tau-q(r) \leqslant 0
$$

where the function $q=q(r)$ is the stress-like softening function, and the equivalent stress, $\tau$, is defined as

$$
\tau=\left\langle t_{\mathrm{n}}\right\rangle
$$

where $t_{\mathrm{n}}$ is the normal stress acting on the plane orthogonal to direction $\mathbf{n}$. The symbols $\langle\cdot\rangle$ are the Macaulay brackets $(\langle x\rangle=x$, if $x \geqslant 0,\langle x\rangle=0$, if $x<0)$.

Variable $r$ is an internal stress-like variable representing the current damage threshold. It depends on the evolution of the effective stresses and its value controls the size of the damage surface. The initial value of the damage threshold is $r_{0}=\sigma_{0}$, where $\sigma_{0}$ is the initial uniaxial damage stress.

In this study, we will use the following exponential softening law:

$$
q(r)=r_{0} \exp \left\{-2 H_{\mathrm{S}}\left(\frac{r-r_{0}}{r_{0}}\right)\right\}, \quad r_{0} \leqslant r
$$

where $H_{\mathrm{S}} \geqslant 0$ is a constant. Figure 5 shows a schematic representation of this function. 


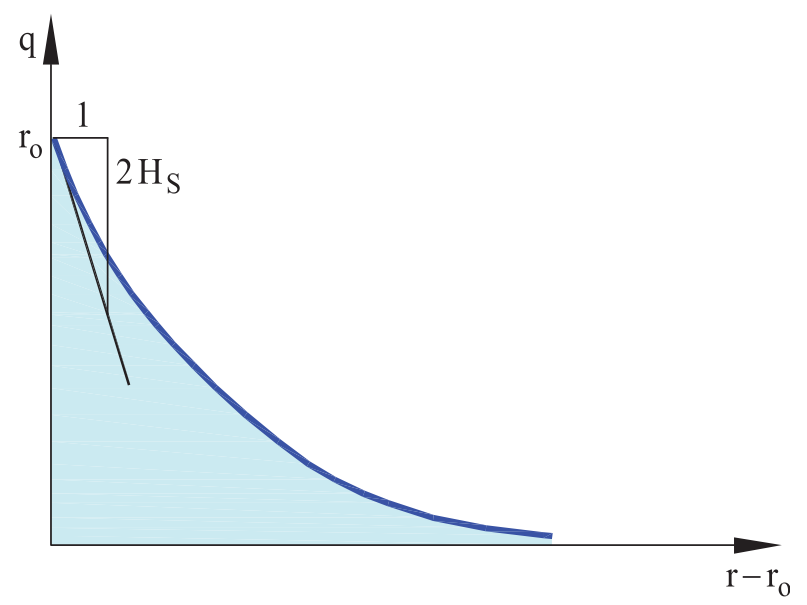

Figure 5. Exponential softening function for the damage model.

The evolution of the damage threshold $r$ and the damage bounding surface for loading, unloading and reloading conditions is controlled by the Kuhn-Tucker relations and the damage consistency condition, which are

$$
\begin{aligned}
& \dot{r} \geqslant 0, \quad \Phi(\tau, r) \leqslant 0, \quad \dot{r} \Phi(\tau, r)=0 \\
& \text { if } \Phi(\tau, r)=0 \quad \text { then } \dot{r} \dot{\Phi}(\tau, r)=0
\end{aligned}
$$

The damage index $d=d(r)$ is explicitly defined in terms of the corresponding current value of the damage threshold

$$
d(r)=1-\frac{q(r)}{r}, \quad r_{0} \leqslant r
$$

so that it is a monotonically increasing function such that $0 \leqslant d \leqslant 1$.

\subsection{Inelastic strain and stress}

Let $\boldsymbol{\varepsilon}$ be the total strain, computed as $\boldsymbol{\varepsilon}=\nabla^{s} \mathbf{u}$, where $\mathbf{u}$ are the displacements, and let us consider the usual decomposition of the total strain tensor into its elastic and inelastic components

$$
\varepsilon=\varepsilon^{\mathrm{e}}+\varepsilon^{\mathrm{i}}
$$

so that we can express

$$
\boldsymbol{\sigma}=\mathbf{C}\left(\boldsymbol{\varepsilon}-\boldsymbol{\varepsilon}^{\mathrm{i}}\right)
$$

As the crucial point to define an orthotropic damage model, let us define the following structure for the inelastic strain:

$$
\varepsilon^{\mathrm{i}}=\mathbf{M}_{\mathrm{e}} \mathbf{e}
$$

where $\mathbf{e}$ is the inelastic deformation vector. Matrix $\mathbf{M}_{\mathrm{e}}$ was introduced in Equation (12). 
Related to the inelastic strain, we can introduce the inelastic stress as

$$
\boldsymbol{\sigma}^{\mathrm{i}}=\mathbf{C} \boldsymbol{\varepsilon}^{\mathrm{i}}=\mathbf{C} \mathbf{M}_{\mathrm{e}} \widehat{\mathbf{C}}^{-1} \mathbf{s}=\mathbf{M}_{\mathrm{s}} \mathbf{s}
$$

where we observe that the structure of the inelastic stress is which is matrix $\mathbf{M}_{\mathrm{s}}$, given by

$$
\mathbf{M}_{\mathrm{s}}=\mathbf{C M}_{\mathrm{e}} \widehat{\mathbf{C}}^{-1}
$$

The stresses in Equation (27) can also be expressed as

$$
\boldsymbol{\sigma}=\overline{\boldsymbol{\sigma}}-\boldsymbol{\sigma}^{\mathrm{i}}
$$

where the effective stress $\overline{\boldsymbol{\sigma}}[30]$ is computed in terms of the total strain $\boldsymbol{\varepsilon}$ as

$$
\overline{\boldsymbol{\sigma}}=\mathbf{C \varepsilon}
$$

\subsection{Traction equilibrium}

Using Equations (14), (27) and (28), we can express

$$
\begin{aligned}
\mathbf{t} & =\mathbf{N}^{\mathrm{T}} \boldsymbol{\sigma} \\
& =\mathbf{N}^{\mathrm{T}} \mathbf{C}\left[\boldsymbol{\varepsilon}-\boldsymbol{\varepsilon}^{\mathrm{i}}\right] \\
& =\overline{\mathbf{t}}-\left[\mathbf{N}^{\mathrm{T}} \mathbf{C} \mathbf{M}_{\mathrm{e}}\right] \mathbf{e}
\end{aligned}
$$

where we have introduced the effective traction vector $\overline{\mathbf{t}}=\mathbf{N}^{\mathrm{T}} \overline{\boldsymbol{\sigma}}$. Substituting law (16) into Equation (33c) leads to

$$
\mathbf{e}=\left[\widehat{\boldsymbol{\Gamma}}^{-1} \widehat{\mathbf{C}}+\mathbf{N}^{\mathrm{T}} \mathbf{C} \mathbf{M}_{\mathbf{e}}\right]^{-1} \overline{\mathbf{t}}=\widehat{\mathbf{D}}_{\mathrm{e}} \overline{\mathbf{t}}
$$

Alternatively, using Equations (29), (33c) can be expressed as

$$
\mathbf{t}=\overline{\mathbf{t}}-\left[\mathbf{N}^{\mathrm{T}} \mathbf{M}_{\mathbf{s}}\right] \mathbf{s}
$$

and, substituting Equation (18), we obtain

$$
\mathbf{s}=\left[\widehat{\boldsymbol{\Gamma}}^{-1}+\mathbf{N}^{\mathrm{T}} \mathbf{M}_{\mathbf{s}}\right]^{-1} \overline{\mathbf{t}}=\widehat{\mathbf{D}}_{\mathbf{s}} \overline{\mathbf{t}}
$$

\section{Remark}

Considering vector $\mathbf{m}$ as $\mathbf{m}=\mathbf{n}+\boldsymbol{\delta}$, where $\boldsymbol{\delta}$ is a mesh-dependent correction to $\mathbf{n}$, we can define the mesh correction projection matrices $\boldsymbol{\Delta}_{\mathrm{e}}=\mathbf{M}_{\mathbf{e}}-\mathbf{N}$ and $\boldsymbol{\Delta}_{\mathrm{s}}=\mathbf{C} \boldsymbol{\Delta}_{\mathrm{e}} \widehat{\mathbf{C}}^{-1}$. Now, assuming that $\widehat{\mathbf{C}}=$ $\mathbf{N}^{\mathrm{T}} \mathbf{C N}$, and operating

$$
\begin{aligned}
\widehat{\mathbf{D}}_{\mathrm{s}} & =\left[\widehat{\boldsymbol{\Gamma}}^{-1}+\mathbf{N}^{\mathrm{T}} \mathbf{M}_{\mathrm{s}}\right]^{-1} \\
& =\left[\widehat{\mathbf{D}}^{-1}+\mathbf{N}^{\mathrm{T}} \boldsymbol{\Delta}_{\mathrm{s}}\right]^{-1} \\
& =\widehat{\mathbf{D}}\left[\mathbf{I}-\mathbf{N}^{\mathrm{T}} \boldsymbol{\Delta}_{\mathrm{s}}\left[\widehat{\mathbf{D}}^{-1}+\mathbf{N}^{\mathrm{T}} \boldsymbol{\Delta}_{\mathrm{s}}\right]^{-1}\right] \\
& =\widehat{\mathbf{D}}-\widehat{\mathbf{D}}_{\Delta}
\end{aligned}
$$

with $\widehat{\mathbf{D}}_{\Delta}=\widehat{\mathbf{D}} \mathbf{N}^{\mathrm{T}} \boldsymbol{\Delta}_{\mathrm{S}}\left[\widehat{\mathbf{D}}^{-1}+\mathbf{N}^{\mathrm{T}} \boldsymbol{\Delta}_{\mathrm{S}}\right]^{-1}$. This expression shows clearly the structure of the nondimensional damage matrix $\widehat{\mathbf{D}}_{\mathrm{S}}$ and the nature of the mesh correction associated with $\boldsymbol{\delta}$. Note that $\widehat{\mathbf{D}}_{\Delta}$ vanishes if $\mathbf{m}=\mathbf{n}$. 
In addition, using Equation (17)

$$
\widehat{\mathbf{D}}_{\mathrm{e}}=\widehat{\mathbf{C}}^{-1}\left[\widehat{\mathbf{D}}-\widehat{\mathbf{D}}_{\Delta}\right]
$$

\subsection{Orthotropic damage}

Once the inelastic deformation vector $\mathbf{e}$ is known from Equation (34), Equation (28) defines the inelastic strain as

$$
\boldsymbol{\varepsilon}^{\mathrm{i}}=\mathbf{M}_{\mathrm{e}} \mathbf{e}=\mathbf{M}_{\mathrm{e}} \widehat{\mathbf{D}}_{\mathrm{e}} \overline{\mathbf{t}}=\left(\mathbf{M}_{\mathrm{e}} \widehat{\mathbf{D}}_{\mathrm{e}} \mathbf{N}^{\mathrm{T}}\right) \overline{\boldsymbol{\sigma}}=\left(\mathbf{M}_{\mathrm{e}} \widehat{\mathbf{D}}_{\mathrm{e}} \mathbf{N}^{\mathrm{T}}\right) \mathbf{C} \boldsymbol{\varepsilon}
$$

On the other hand, the inelastic stress is, from Equations (36) and (29)

$$
\boldsymbol{\sigma}^{\mathrm{i}}=\mathbf{M}_{\mathrm{s}} \mathbf{s}=\mathbf{M}_{\mathrm{s}} \widehat{\mathbf{D}}_{\mathrm{s}} \overline{\mathbf{t}}=\left(\mathbf{M}_{\mathrm{s}} \widehat{\mathbf{D}}_{\mathrm{s}} \mathbf{N}^{\mathrm{T}}\right) \overline{\boldsymbol{\sigma}}=\mathbf{D} \overline{\boldsymbol{\sigma}}
$$

so that the orthotropic damage matrix can be expressed as

$$
\mathbf{D}=\mathbf{M}_{\mathrm{s}} \widehat{\mathbf{D}}_{\mathrm{s}} \mathbf{N}^{\mathrm{T}}
$$

or, alternatively, as

$$
\mathbf{D}=\mathbf{C}\left(\mathbf{M}_{\mathrm{e}} \widehat{\mathbf{D}}_{\mathrm{e}} \mathbf{N}^{\mathrm{T}}\right)
$$

Finally, from Equations (31) and (40), the constitutive equation for the orthotropic damage model is defined as

$$
\boldsymbol{\sigma}=(\mathbf{I}-\mathbf{D}) \overline{\boldsymbol{\sigma}}=(\mathbf{I}-\mathbf{D}) \mathbf{C} \boldsymbol{\varepsilon}
$$

where $\mathbf{I}$ is the identity matrix and $\mathbf{D}$ is the orthotropic damage matrix.

Because the value of the current normal stress in criterion (21) depends on the damage matrix and this, in turn, depends on the damage index, the evaluation of the current stress is a non-linear procedure that must be solved iteratively. The corresponding algorithm is described in Figure 6. There, superindex $(k)$ refers to the iteration counter of the non-linear loop that updates the damage index and the other inelastic entities.

\begin{tabular}{|r|l|l|}
\hline 1. & Compute effec. stress \& traction: & $\overline{\boldsymbol{\sigma}}=\mathbf{C} \boldsymbol{\varepsilon} ; \quad \overline{\mathbf{t}}=\mathbf{N}^{\mathrm{T}} \overline{\boldsymbol{\sigma}}$ \\
\hline 2. & Initialize iteration counter: & $k=0$ \\
\hline 3. & Initialize damage threshold: & $r^{(k)}=\left\langle\bar{t}_{n}^{(k)}\right\rangle$ \\
\hline 4. & Update iteration counter: & $k=k+1$ \\
\hline 5. & Update damage index: & $d^{(k)}=1-\left(q\left(r^{(k)}\right) / r^{(k)}\right)$ \\
\hline 6. & Compute damage matrix: & $\mathbf{D}^{(k)}=\mathbf{M}_{\mathbf{s}} \widehat{\mathbf{D}}_{\mathbf{s}}^{(k)} \mathbf{N}^{\mathrm{T}}$ \\
\hline 7. & Compute stress \& traction: & $\boldsymbol{\sigma}^{(k)}=\left(\mathbf{I}-\mathbf{D}^{(k)}\right) \overline{\boldsymbol{\sigma}} ; \quad \mathbf{t}^{(k)}=\mathbf{N}^{\mathrm{T}} \boldsymbol{\sigma}^{(k)}$ \\
\hline 8. & Compute equivalent stress: & $\tau^{(k)}=\left\langle t_{n}^{(k)}\right\rangle$ \\
\hline 9. & Update damage threshold: & $r^{(k)}=\tau^{(k)} /\left(1-d^{(k)}\right)$ \\
\hline 10. & Check convergence on $r^{(k)}$ & \\
\hline 11. & If NOT converged: & $\mathrm{GO} \mathrm{TO} \mathrm{Step} 4$. \\
\hline 12. & If converged: & SET $r=r^{(k)}$ and $d=d^{(k)}$ \\
\hline
\end{tabular}

Figure 6. Algorithm for the determination of the damage index. 


\subsection{Mechanical dissipation}

In the continuum case, when $\boldsymbol{\delta}=\mathbf{0}$ and $\mathbf{m}=\mathbf{n}$, damage matrix $\widehat{\mathbf{D}}_{\mathrm{e}}$ is symmetric and the constitutive model can be appropriately derived from the mechanical free energy, defined as

$$
W=\frac{1}{2} \boldsymbol{\varepsilon}^{\mathrm{T}}(\mathbf{I}-\mathbf{D}) \mathbf{C} \boldsymbol{\varepsilon} \geqslant 0
$$

The necessary condition $W \geqslant 0$ is verified if $\mathbf{D}$ is positive definite and $\|\mathbf{D}\| \leqslant 1$. These conditions verify if matrix $\widehat{\mathbf{D}}_{\mathrm{e}}$ is positive definite and $\left\|\widehat{\mathbf{D}}_{\mathrm{e}}\right\| \leqslant 1$. From Equations (20) and (34), and taking $\widehat{\mathbf{C}}=\mathbf{N}^{\mathrm{T}} \mathbf{C N}$, it is

$$
\widehat{\mathbf{D}}_{\mathrm{e}}=\widehat{\mathbf{C}}^{-1} \widehat{\mathbf{D}}
$$

so $\widehat{\mathbf{D}}$ must be positive definite and $\|\widehat{\mathbf{D}}\| \leqslant 1$.

Applying Coleman's method, the secant constitutive equation (43) can be obtained from Equation (44). By the same procedure, the rate of mechanical dissipation is obtained as

$$
\dot{\mathscr{D}}=\frac{1}{2} \boldsymbol{\varepsilon}^{\mathrm{T}} \dot{\mathbf{D}} \mathbf{C} \boldsymbol{\varepsilon} \geqslant 0
$$

The condition $\dot{\mathscr{D}} \geqslant 0$ is verified provided that the rate of the damage matrix $\dot{\mathbf{D}}$ is positive definite. In view of Equations (42) and (45), this reduces to the condition that the rate of the traction damage matrix $\dot{\hat{\mathbf{D}}}$ must be positive definite.

The necessary conditions on $\widehat{\mathbf{D}}$ and $\widehat{\mathbf{D}}$ depend on the actual definition of $\widehat{\mathbf{D}}$. For the simple case $\widehat{\mathbf{D}}=d \mathbf{I}$, it is $\hat{\mathbf{D}}=\dot{d} \mathbf{I}$ and, therefore, the conditions reduce to the standard inequalities $0 \leqslant d \leqslant 1$ and $\dot{d} \geqslant 0$.

Using the definition of the effective traction vector $\overline{\mathbf{t}}=\mathbf{N}^{\mathrm{T}} \overline{\boldsymbol{\sigma}}$ and with some manipulation, Equation (46) can be rewritten as

$$
\dot{\mathscr{D}}=\frac{1}{2} \overline{\mathbf{t}}^{\mathrm{T}} \widehat{\mathbf{C}}^{-1} \hat{\mathbf{D}} \overline{\mathbf{t}} \geqslant 0
$$

which shows that all dissipation occurs on the damaged plane.

In the discrete case, when $\boldsymbol{\delta} \neq \mathbf{0}$ and $\mathbf{m} \simeq \mathbf{n}$, matrix $\widehat{\mathbf{D}}_{\mathrm{e}}$ is non-symmetric and the mechanical free energy cannot be defined. Without resorting to a full convergence analysis, but in the spirit of all discretization methods, we will assume that the discrete counterparts of Equations (43) and (46) will converge to the continuum values on mesh refinement, in a global sense. This does not require that $\boldsymbol{\delta} \rightarrow \mathbf{0}$ pointwise. This convergence argument is equally applicable to the discrete implementation of the non-symmetric strong discontinuity approach, sometimes accused of thermodynamical inconsistency because of its non-symmetric format.

\subsection{Strain-softening and fracture energy release}

Let us now consider an uniaxial tensile experiment such as the one described in Section 2.2, in which the strain increases monotonically and quasi-statically from an initial unstressed state to another in which full degradation takes place. Without loss of generality, we will assume $v=0$. In this case

$$
\overline{\mathbf{t}}=\frac{t_{\mathrm{n}}}{1-d} \mathbf{n}, \quad \widehat{\mathbf{C}}=\left[\begin{array}{ll}
E & 0 \\
0 & G
\end{array}\right], \quad \hat{\mathbf{D}}=\dot{d} \mathbf{I}
$$


Substituting these in Equation (47), and noting that $q=t_{\mathrm{n}}$ and $r=t_{\mathrm{n}} /(1-d)$, the specific energy dissipated in the process is

$$
\mathscr{D}=\int_{t=0}^{t=\infty} \dot{\mathscr{D}} \mathrm{d} t=\int_{t=0}^{t=\infty} \frac{\dot{d}}{1-d} \frac{t_{\mathrm{n}}^{2}}{E} \mathrm{~d} t=\frac{1}{E} \int_{r=r_{0}}^{r=\infty} q \mathrm{~d} r=\left(1+\frac{1}{H_{\mathrm{S}}}\right) \frac{\sigma_{0}^{2}}{2 E}
$$

In order to relate the specific dissipated energy $\mathscr{D}$, defined per unit volume in the localization band, to the mode I fracture energy of the material $\mathscr{G}_{\mathrm{f}}$, defined per unit area of damaged material, we introduce the characteristic length $h$, width of the localization band, so that

$$
\mathscr{D} h=\mathscr{G}_{\mathrm{f}}
$$

Now, equating $\mathscr{D}=\mathscr{G}_{\mathrm{f}} / h$ to the result in Equation (49), we have

$$
H_{\mathrm{S}}=\frac{\bar{H}_{\mathrm{S}} h}{1-\bar{H}_{\mathrm{S}} h} \geqslant 0
$$

where $\bar{H}_{\mathrm{S}}=\sigma_{0}^{2} /\left(2 E \mathscr{G}_{\mathrm{f}}\right)$ depends only on the material properties. This procedure makes the softening modulus $H_{\mathrm{S}}$, which defines the softening response, dependent on length $h$. Defining the material length $\overline{l_{\mathrm{S}}}=1 / \bar{H}_{\mathrm{S}}$, Equation (51) can be rewritten as

$$
H_{\mathrm{S}}=\frac{h}{\overline{l_{\mathrm{S}}}-h} \simeq \frac{h}{\overline{l_{\mathrm{S}}}}
$$

where the approximation holds for $\overline{l_{\mathrm{S}}} \gg h$.

\section{THEORETICAL ANALYSIS}

In this section we show that the smeared-embedded crack model defined above is able to reproduce properly the expected strain and stress states in a situation of progressive uniaxial straining with localization as the one described in Section 2.2. From there and Figure 3, let us recall that the discrete total strain is given by Equation (9) as

$$
\left[\begin{array}{c}
\varepsilon_{x} \\
\varepsilon_{y} \\
\gamma_{x y}
\end{array}\right]=\left[\begin{array}{c}
1 \\
-v(1-\beta) \\
m \beta
\end{array}\right]\left(\overline{\varepsilon_{x}}\right)
$$

where $m=m_{y} / m_{x}$ is the tangent of the angle between vectors $\mathbf{m}$ and $\mathbf{n}$ and $\beta=\left(\overline{\varepsilon_{x}}\right)^{\mathrm{i}} /\left(\overline{\varepsilon_{x}}\right)$ is the ratio of the inelastic to the total strain in the $x$-direction. This ratio is to be determined, as it is obviously related to the damage index.

Assuming plane stress conditions, the isotropic linear-elastic constitutive matrix of the undamaged material is

$$
\mathbf{C}=\frac{E}{1-v^{2}}\left[\begin{array}{ccc}
1 & v & 0 \\
v & 1 & 0 \\
0 & 0 & \frac{2}{1-v}
\end{array}\right]
$$


where $E$ and $v$ are Young's modulus and the Poisson ratio, respectively. The shear modulus is $G=E / 2(1+v)$.

Assuming, as in Figure 3, that vector $\mathbf{n}=[1,0]^{\mathrm{T}}$ and vector $\mathbf{m}=\left[m_{x}, m_{y}\right]^{\mathrm{T}}$, we have

$$
\mathbf{N}=\left[\begin{array}{ll}
1 & 0 \\
0 & 0 \\
0 & 1
\end{array}\right], \quad \mathbf{M}_{\mathbf{e}}=\left[\begin{array}{cc}
m_{x} & 0 \\
0 & m_{y} \\
m_{y} & m_{x}
\end{array}\right], \quad \mathbf{M}_{\mathrm{s}}=\left[\begin{array}{cc}
m_{x} & \frac{2 v}{1-v} m_{y} \\
v m_{x} & \frac{2}{1-v} m_{y} \\
\frac{1-v}{2} m_{y} & m_{x}
\end{array}\right]
$$

and

$$
\widehat{\mathbf{C}}=\mathbf{N}^{\mathrm{T}} \mathbf{C N}=\frac{E}{1-v^{2}}\left[\begin{array}{cc}
1 & 0 \\
0 & \frac{2}{1-v}
\end{array}\right]
$$

Given the total strain, the effective stress is

$$
\overline{\boldsymbol{\sigma}}=\mathbf{C} \boldsymbol{\varepsilon}=\frac{E}{1-v^{2}}\left[\begin{array}{c}
1-v^{2}(1-\beta) \\
v \beta \\
\left(\frac{1-v}{2}\right) m \beta
\end{array}\right]\left(\overline{\varepsilon_{x}}\right)
$$

Therefore, the effective traction is

$$
\overline{\mathbf{t}}=\mathbf{N}^{\mathrm{T}} \overline{\boldsymbol{\sigma}}=\frac{E}{1-v^{2}}\left[\begin{array}{c}
1-v^{2}(1-\beta) \\
\left(\frac{1-v}{2}\right) m \beta
\end{array}\right]\left(\overline{\varepsilon_{x}}\right)
$$

Assuming a diagonal form for the traction damage matrix $\widehat{\mathbf{D}}=d \mathbf{I}$, we have

$$
\begin{aligned}
& \widehat{\mathbf{D}}_{\mathrm{s}}=\left[\widehat{\boldsymbol{\Gamma}}^{-1}+\mathbf{N}^{\mathrm{T}} \mathbf{M}_{\mathrm{s}}\right]^{-1} \\
& =\frac{\tilde{d}}{m_{x}}\left[\begin{array}{cc}
1 & -v m \tilde{d} \\
\left(\frac{1-v}{2}\right) m \tilde{d} & 1
\end{array}\right]^{-1} \\
& =\frac{\tilde{d} / m_{x}}{1-v(m \widetilde{d})^{2}}\left[\begin{array}{cc}
1 & -\left(\frac{2 v}{1-v}\right) m \tilde{d} \\
-\left(\frac{1-v}{2}\right) m \tilde{d} & 1
\end{array}\right]
\end{aligned}
$$


where $\widetilde{d}=m_{x} d /\left[1-d\left(1-m_{x}\right)\right]$. Therefore,

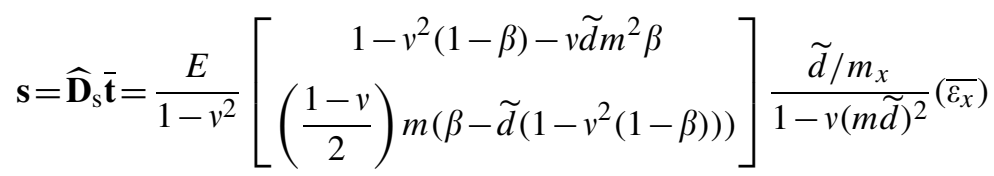

From this, we can identify $\beta=\left(\overline{\varepsilon_{x}}\right)^{\mathrm{i}} /\left(\overline{\varepsilon_{x}}\right)=\widetilde{d}\left(1-v^{2}\right) /\left(1-v^{2} \widetilde{d}\right)$, and rewrite, in a much simpler way

$$
\mathbf{s}=\frac{E}{1-v^{2}}\left[\begin{array}{l}
1 \\
0
\end{array}\right] \frac{\beta}{m_{x}}\left(\overline{\varepsilon_{x}}\right)
$$

Now, constructing the inelastic stress, we have

$$
\boldsymbol{\sigma}^{\mathrm{i}}=\mathbf{M}_{\mathrm{s}} \mathbf{s}=\frac{E}{1-v^{2}}\left[\begin{array}{c}
\beta \\
v \beta \\
\left(\frac{1-v}{2}\right) m \beta
\end{array}\right]\left(\overline{\varepsilon_{x}}\right)
$$

Finally, subtracting the inelastic stress from the effective stress, we have the correct expression for the stress, given by

$$
\boldsymbol{\sigma}=\overline{\boldsymbol{\sigma}}-\boldsymbol{\sigma}^{\mathrm{i}}=\left[\begin{array}{c}
(1-\beta) E \\
0 \\
0
\end{array}\right]\left(\overline{\varepsilon_{x}}\right)
$$

where it can be observed that the spurious shear has been completely removed.

Note that at the inception of damage, when $d \ll 1$, the first term in Equation (59a) is dominant, $\widetilde{d} \simeq m_{x} d$ and, therefore, the evolution of the damage index is affected by the direction of vector $\mathbf{m}$. On the other hand, when damage is nearly complete, that is, $d \rightarrow 1, \beta \rightarrow 1$, the relaxation of the axial stress is complete.

We can compare this result with the one obtained with a classical orthotropic damage model. To that end, we will consider that in this case

$$
\mathbf{M}_{\mathrm{s}}=\mathbf{C N \widehat { C } ^ { - 1 }}=\left[\begin{array}{ll}
1 & 0 \\
v & 0 \\
0 & 1
\end{array}\right]
$$

and

$$
\widehat{\mathbf{D}}_{\mathrm{s}}=\left[\widehat{\boldsymbol{\Gamma}}^{-1}+\mathbf{N}^{\mathrm{T}} \mathbf{M}_{\mathrm{s}}\right]^{-1}=\widehat{\mathbf{D}}=d \mathbf{I}
$$

Therefore,

$$
\mathbf{s}=\widehat{\mathbf{D}}_{\mathrm{s}} \overline{\mathbf{t}}=\frac{E}{1-v^{2}}\left[\begin{array}{c}
1-v^{2}(1-\beta) \\
\left(\frac{1-v}{2}\right) m \beta
\end{array}\right] d\left(\overline{\varepsilon_{x}}\right)
$$


From this, we can identify $\beta=\left(\overline{\varepsilon_{x}}\right)^{\mathrm{i}} /\left(\overline{\varepsilon_{x}}\right)=d\left(1-v^{2}\right) /\left(1-v^{2} d\right)$ and rewrite

$$
\mathbf{s}=\frac{E}{1-v^{2}}\left[\left(\frac{1-v}{2}\right) m d\right] \beta\left(\overline{\varepsilon_{x}}\right)
$$

Now, constructing the inelastic stress, we have

$$
\boldsymbol{\sigma}^{\mathrm{i}}=\mathbf{M}_{\mathrm{s}} \mathbf{s}=\frac{E}{1-v^{2}}\left[\begin{array}{c}
\beta \\
v \beta \\
\left(\frac{1-v}{2}\right) m d \beta
\end{array}\right]\left(\overline{\varepsilon_{x}}\right)
$$

Finally, subtracting the inelastic stress from the effective stress, we have the stress given by

$$
\boldsymbol{\sigma}=\overline{\boldsymbol{\sigma}}-\boldsymbol{\sigma}^{\mathrm{i}}=\left[\begin{array}{c}
(1-\beta) E \\
0 \\
m(1-d) \beta G
\end{array}\right]\left(\overline{\varepsilon_{x}}\right)
$$

where it can be observed that the spurious shear strain produced by the misalignment of the crack with respect to the sides of the FE transforms into a corresponding spurious shear stress.

Figure 4 (right) represents the evolution of the axial and shear stress components in the 'classical' orthotropic model as the axial strain increases. The assumed material properties are Young's modulus $E=2 \mathrm{GPa}$, the Poisson ratio $v=0.3$, tensile strength $\sigma_{0}=1 \mathrm{MPa}$ and mode I fracture energy $G_{\mathrm{f}}=250 \mathrm{~J} / \mathrm{m}^{2}$. The angle between vectors $\mathbf{n}$ and $\mathbf{m}$ is assumed to be $\alpha=\arctan m=-30^{\circ}$, $m=-\sqrt{3} / 3$. Note how the axial stress reaches the peak and reduces exponentially thereafter. On the other hand, the shear stress, which is null prior to cracking, increases after cracking to reach a maximum value that is about $15 \%$ of the tensile strength. This spurious shear stress is progressively released as damage increases. Figure 4 (right) also represents the evolution of the axial and shear stress components in the corresponding 'mesh-corrected' orthotropic model. Note that in this case the shear stress remains null throughout the loading process.

\section{NUMERICAL EXAMPLES}

The formulation presented in the preceding sections is illustrated below by solving two different benchmark problems. The performance of the standard continuous displacement FEs is tested considering $2 D$ plane-strain 3-noded linear triangular meshes.

The examples are solved using the orthotropic damage model presented in Section 3 with exponential softening, adjusted according to the element size. The following material properties are assumed for both examples: Young's modulus $E=30 \mathrm{GPa}$, the Poisson ratio $v=0.2$, tensile strength $\sigma_{0}=2 \mathrm{MPa}$ and mode I fracture energy $G_{\mathrm{f}}=100 \mathrm{~J} / \mathrm{m}^{2}$.

The discrete problem is solved incrementally, in a (pseudo)time step-by-step manner. In all cases 200 equal time steps are performed to complete the analyses. Within each step, convergence of 
the non-linear procedure is attained when the ratio of the norm of the iterative to the incremental norm of the computed displacements is lower than $10^{-4}$.

In the following examples, the determination of the direction of propagation of the strain localization band is treated as a separate problem, coupled to that of solving the balance of momentum equation. The use of crack tracking algorithms is a well-established practice in fracture mechanics and also in continuum mechanics when embedded formulations are used. The convenience of using these techniques in the context of smeared models is justified in References [26, 27] from the stability analysis of the weak form of the associated discrete mechanical problem. In all the examples, the smearing distance, $h$, is computed as explained in Section 2.2.

Calculations are performed with an enhanced version of the FE program COMET [31], developed at the International Center for Numerical Methods in Engineering (CIMNE). Pre- and postprocessing is done with $\mathrm{GiD}$, also developed at CIMNE [32].

\subsection{Perforated strip under tension}

The first example is a plane-strain perforated strip subjected to axial vertical straining imposed at both ends. Because of the symmetry of the domain and boundary conditions, only one half of the domain (the right half) is considered. Dimensions of the strip are $20 \times 40 \mathrm{~cm} \times \mathrm{cm}$ (width $\times$ height) and the radius of the perforation is $r=1 \mathrm{~cm}$. This example is selected because the initial geometry does not present any singular point; tensile stresses are larger in the vicinity of the perforation and damage starts there. It also represents an example of pure mode I fracture.

The computational domain is discretized in two different unstructured meshes with average mesh sizes of $h_{\mathrm{e}}=5 \mathrm{~mm}$ (2023 nodes) and $h_{\mathrm{e}}=2.5 \mathrm{~mm}$ (7648 nodes). The central part of the two meshes is shown in Figure 7. The pre-processor used tends to introduce patches of equilateral triangles with predominant directions at $-30,+30$ and $+90^{\circ}$ with the horizontal axis, none of them parallel to the expected crack, which must propagate along the horizontal symmetry axis. It must be remarked that, because both meshes are rather fine and there are no singular strain/stress points in the domain, the difference in the slope of the elastic response of both meshes is less than $0.05 \%$ (see Figure 8 ).

The computed deformed shapes of the strip in the vicinity of the perforation are shown in Figures 7(a) and (b) for the coarse and fine meshes, respectively ((half)-imposed vertical displacement $\delta=0.1 \mathrm{~mm}$, with a displacement amplification factor of 100 ; the other half-imposed displacement is applied at the opposite end of the strip). The different element sizes in both

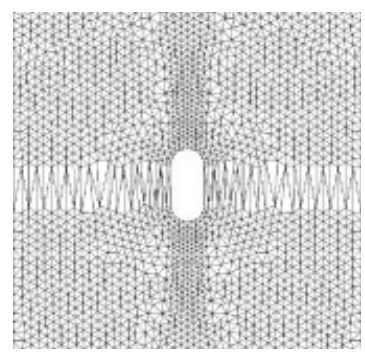

(a)

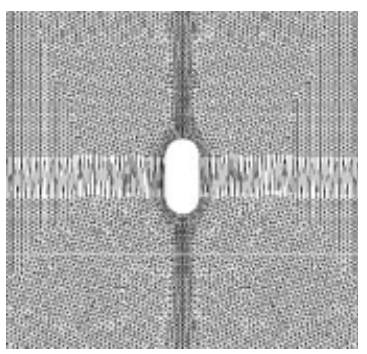

(b)

Figure 7. Deformed geometries $(\times 100)$ on the central part of the specimen for perforated strip: (a) coarse mesh and (b) fine mesh. 


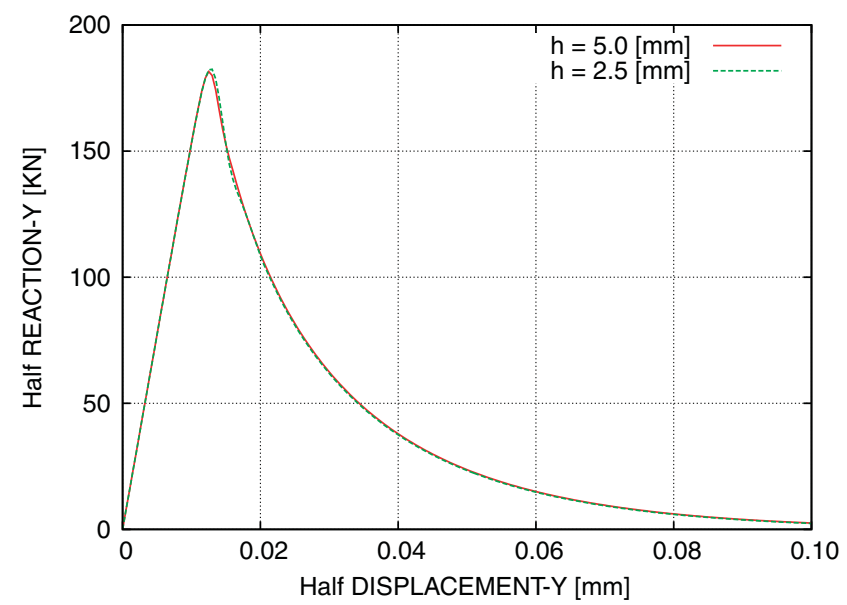

Figure 8. Load vs displacement for perforated strip. Comparison between different mesh sizes.

meshes can be appreciated in these figures. As shown, the tracking technique used ensures that the computed cracks in both analyses follow exactly the horizontal axis of symmetry of the perforation, even if the elements in neither of the two meshes are aligned along this line.

Figure 8 shows (half)-load vs (half)-imposed vertical displacement curves obtained in the two analyses. Because in this example the strain field is almost uniform prior to the inception of the cracks, the response curve is almost linear until the cracks form in a rather explosive manner, with a nearly exponential softening branch after the limit load is attained.

Note that the overall global response is satisfactorily similar upon mesh refinement, with the total area under the load-displacement curve converging to the correct amount of energy dissipated to create the cracks. No spurious brittleness is observed when the size of the elements is reduced.

Figure 9 shows the results obtained using the proposed formulation on the fine mesh. The three columns represent, at the inception of damage (top) and when damage is almost complete (bottom): (a) the contours for the vertical displacements, (b) the contours for the maximum principal strain and (c) maximum principal strain vectors. The progressive concentration of the displacement gradients (strains) in the elements lying along the horizontal axis of symmetry is evident in the three columns. The bottom figures show how, when the failure mechanism is fully developed, all the deformation concentrates in the formed horizontal crack, while the elements outside this localization band are mostly undeformed. Therefore, the resolution of the cracks is optimal for the mesh used. In the third column, it can be observed that the correct failure mechanism has been predicted although the directions of the computed maximum principal total strain vectors are clearly dependent on the mesh bias, as they are not vertical everywhere. For the coarser mesh, similar results are obtained, although the strain localization is smeared across a row of elements twice as large.

\subsection{Four-point bending beam}

The second example is a plane-strain doubly notched beam subjected to four-point bending. Figure 10 depicts the geometry of the problem; dimensions of the beam are $134.0 \times 30.6 \mathrm{~cm} \times \mathrm{cm}$ 

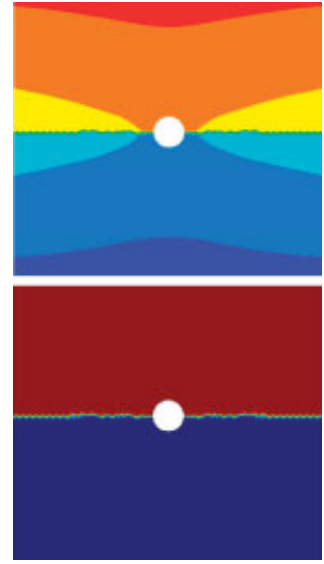

(a)
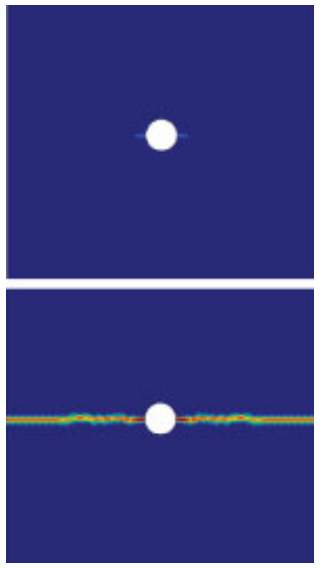

(b)

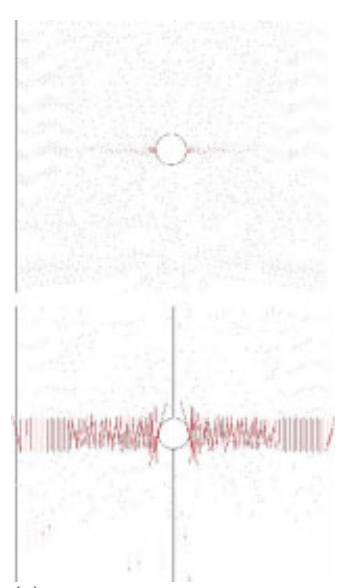

(c)

Figure 9. Results for perforated strip using the proposed formulation. Evolution of (a) vertical displacement, (b) maximum principal total strain and (c) vectors of maximum principal total strain.

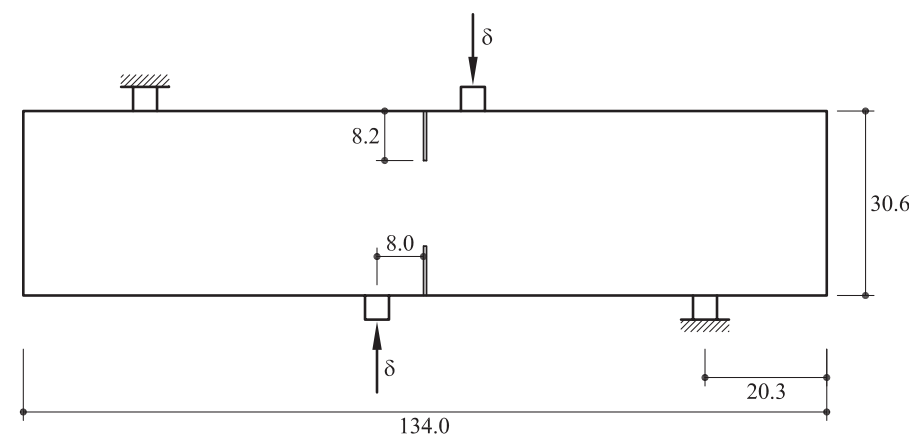

Figure 10. Geometry and load for four-point bending beam.

(width $\times$ height) and the length and width of the notches are 8.2 and $0.5 \mathrm{~cm}$, respectively. The load is applied at the central (rigid) supports (at $8.0 \mathrm{~cm}$ from the centre of the beam) by imposing vertical displacements of opposite sign at the top and bottom supports. The two supports near the extremes of the beam (at $20.3 \mathrm{~cm}$ ) are fixed. This example is selected because it presents two singular points at the tips of the notches; tensile stresses are very large in the vicinity of these regions and damage starts there. It also represents an excellent example of mixed mode fracture.

The computational domain is discretized in three different unstructured meshes with average mesh sizes of $h_{\mathrm{e}}=20 \mathrm{~mm}$ (1189 nodes), $h_{\mathrm{e}}=10 \mathrm{~mm}$ ( 2217 nodes) and $h_{\mathrm{e}}=5 \mathrm{~mm}$ (5909 nodes). The central part of the three meshes is shown in Figure 11. Although all meshes are rather fine, the coarse and medium meshes exhibit a slope of the elastic response that is 4.34 and $2.10 \%$ stiffer than the fine mesh, respectively (see Figure 12). This difference is due to the presence of singular strain/stress points in the domain, and it affects appreciably the linear and non-linear response of the three meshes. 
The computed deformed shapes of the central part of the beam, using the three different meshes, are shown in Figures 11(a)-(c), respectively (imposed vertical displacement $\delta=0.1 \mathrm{~mm}$, with a displacement amplification factor of 100). The different element sizes in the meshes can be appreciated in these figures. As shown, the computed cracks in all the analyses follow very closely the same path, starting at the tip of the notches and turning upwards to the point of application of the loads. No spurious mesh bias is observed in any of the meshes.

Figure 12 shows load vs imposed vertical displacement curves obtained in the three analyses. In this example the loading branch curves slowly as the cracks progress, turning into the softening branch once the failure mechanism is fully developed. The load does not vanish completely because only damage due to tensile effective stresses is considered, and the state of stresses near the loading supports is mostly compressive. The overall global response is very similar upon mesh refinement, although the effect of the different spatial discretizations can be observed. For comparison, Figure 12 also shows the curve obtained using a 'classical' orthotropic damage without mesh correction (medium mesh, $h_{\mathrm{e}}=10 \mathrm{~mm}$ ). In this case, the spurious shear stresses arrest the developing cracks

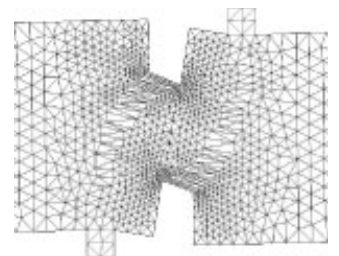

(a)

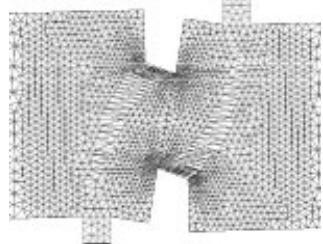

(b)

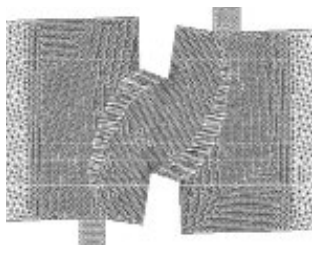

(c)

Figure 11. Deformed geometries $(\times 100)$ on the three meshes for four-point bending beam: (a) coarse; (b) medium; and (c) fine.

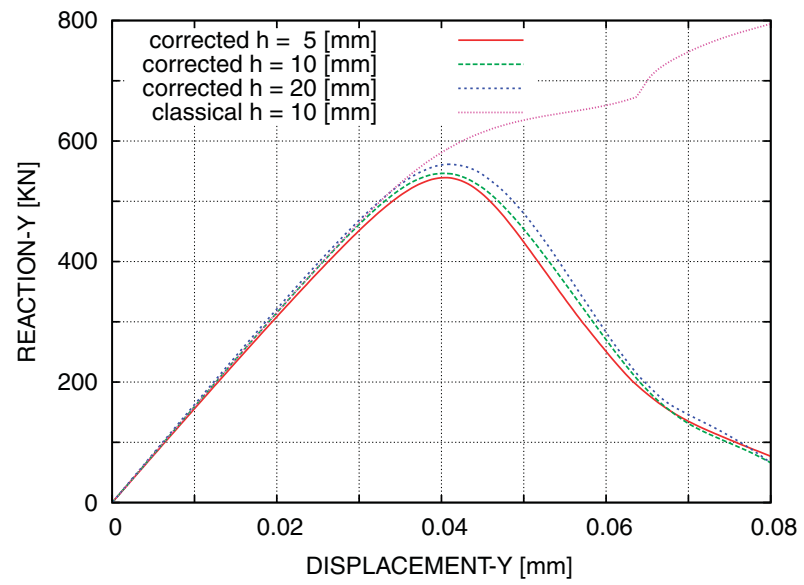

Figure 12. Load vs displacement for four-point bending beam with 'corrected' and 'classical' orthotropic models. 

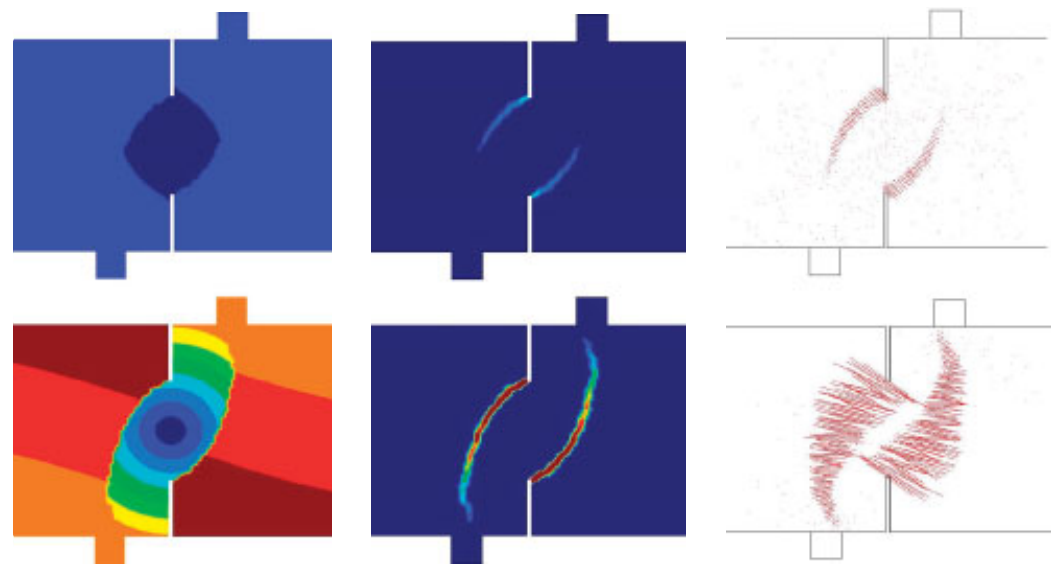

(a)

(b)

(c)

Figure 13. Results for four-point bending beam using the proposed formulation. Contours of (a) displacement, (b) maximum principal total strain, and (c) vectors of maximum principal total strain.

when they reach the central axis of the beam, approximately, and these cannot progress further. This shows in the diagram, as stress locking does not allow the reactions at the supports to reduce after the peak values.

Figure 13 shows the results obtained using the proposed formulation on the fine mesh. The three columns represent, at the inception of damage (top) and when damage is almost complete (bottom) (a) the contours for the total displacements, (b) the contours for the maximum principal strain and (c) the maximum principal strain vectors. As in the previous example, the bottom figures show how, when the failure mechanism is fully developed, all the deformation concentrates in the formed cracks, whereas the elements outside these bands are mostly undeformed. In the third column, it can be observed that the correct failure mechanism has been predicted although the directions of the computed maximum principal total strain vectors are clearly dependent on the mesh bias, as they are not orthogonal to the crack path everywhere. Note in the left bottom plot how, once both cracks are formed, the central part of the beam rotates almost as a rigid body around the centre of the beam.

For the coarser meshes, similar results are obtained, although the strain localization is smeared across a row of larger elements (see Figure 11).

\section{CONCLUSIONS}

The objective of this paper is to formulate a crack model in the spirit of the original continuous crack approaches, where displacement jumps across the crack are smeared over the affected elements and the behaviour of the crack is established through a softening stress-(total) strain law, using standard FEs, such as linear triangles, and local constitutive models, such as orthotropic continuum damage models. This approach is termed as embedded-smeared mesh-corrected crack model. 
The shear locking observed in the discrete problem when the classical methods are used is overcome with an idea borrowed from the well-established discontinuous embedded formulations: the structure of the inelastic strains is linked to the geometry of the cracked element. The resulting formulation is easily implemented in standard non-linear FE codes and it is suitable for engineering applications in 2D and 3D. It is analytically demonstrated that the use of the proposed mesh corrected crack model eliminates the stress locking commonly associated with orthotropic crack models.

Numerical examples show that the smeared models, used in conjunction with appropriate tracking techniques, do not suffer from spurious dependence of the computed structural response on the mesh directional alignment. In addition, computed solutions indicate that continuous displacement interpolations can reproduce problems involving crack propagation as satisfactorily as the discontinuous approaches, if the softening parameter of the constitutive model is properly related to the fracture energy of the material and to the size of the FEs in the localization band.

\section{ACKNOWLEDGEMENTS}

The help of Prof R. Codina is gratefully acknowledged. Financial support from the Spanish Ministry for Education and Science under the SEDUREC project (CSD2006-00060) is also acknowledged.

\section{REFERENCES}

1. Ngo D, Scordelis AC. Finite element analysis of reinforced concrete beams. ACI Journal 1967; 64(14):152-163.

2. Rashid Y. Analysis of prestressed concrete pressure vessels. Nuclear Engineering and Design 1968; 7:334-344.

3. Belytschko T, Black T. Elastic crack growth in finite elements with minimal remeshing. Computer Methods in Applied Mechanics and Engineering 1999; 45(5):601-620.

4. Möes N, Dolbow J, Belytschko T. A finite element method for crack growth without remeshing. International Journal for Numerical Methods in Engineering 1999; 46:131-150.

5. Sukumar N, Möes N, Moran B, Belytschko T. Extended finite element method for three-dimensional crack modelling. International Journal for Numerical Methods in Engineering 2000; 48:1549-1570.

6. Simo JC, Oliver J, Armero F. An analysis of strong discontinuities induced by strain-softening in rate-independent inelastic solids. Computational Mechanics 1993; 12:49-61.

7. Oliver J. Continuum modeling of strong discontinuities in solid mechanics using damage models. Computational Mechanics 1995; 17:277-296.

8. Oliver J, Cervera M, Manzoli O. Strong discontinuities and continuum plasticity models: the strong discontinuity approach. International Journal of Plasticity 1999; 15:319-351.

9. Jirásek M. Comparative study on finite elements with embedded discontinuities. Computer Methods in Applied Mechanics and Engineering 2000; 188:307-330.

10. Jirásek M. Embedded crack model: I. Formulation. International Journal for Numerical Methods in Engineering 2001; 50:1269-1290.

11. Jirásek M. Embedded crack model: II: Combination with smeared cracks. International Journal for Numerical Methods in Engineering 2001; 50:1291-1305.

12. Mosler J, Meschke G. Embedded crack vs. smeared crack models: a comparison of elementwise discontinuous crack path approaches with emphasis on mesh bias. Computer Methods in Applied Mechanics and Engineering 2004; 193:3351-3375.

13. Manzoli OL, Shing PB. A general technique to embed non-uniform discontinuities into standard solid finite elements. Computers and Structures 2006; 84:742-757.

14. Linder C, Armero F. Finite elements with embedded strong discontinuities for the modeling of failure in solids. International Journal for Numerical Methods in Engineering 2007; 72:1391-1433.

15. Sancho JM, Planas J, Cendón DA, Reyes E, Gálvez JC. An embedded cohesive crack model for finite element analysis of concrete fracture. Engineering Fracture Mechanics 2007; 74(1-2):75-86. 
16. Sancho JM, Planas J, Fathy AM, Gálvez JC, Cendón DA. Three-dimensional simulation of concrete fracture using embedded crack elements without enforcing crack path continuity. International Journal for Numerical and Analytical Methods in Geomechanics 2007; 31(2):173-187.

17. Oliver J, Cervera M, Oller S, Lubliner L. Isotropic damage models and smeared crack analysis of concrete. Proceedings of the II International Conference on Computer Aided Analysis and Design of Concrete Structures, SCI-C'90, Zell Am See, Austria, 1990.

18. Cervera M, Oliver J, Oller S, Galindo M. Pathological behaviour of large concrete dams analysed via isotropic damage models. Proceedings of the II International Conference on Computer Aided Analysis and Design of Concrete Structures, SCI-C'90, Zell Am See, Austria, 1990.

19. Jirásek M, Zimmermann T. Analysis of rotating crack model. Journal of Engineering Mechanics (ASCE) 1998; 124(8):842-851.

20. Cervera M, Chiumenti M, Agelet de Saracibar C. Softening, localization and stabilization: capture of discontinuous solutions in J2 plasticity. International Journal for Numerical and Analytical Methods in Geomechanics 2003; 28:373-393.

21. Cervera M, Chiumenti M, Agelet de Saracibar C. Shear band localization via local $J_{2}$ continuum damage mechanics. Computer Methods in Applied Mechanics and Engineering 2003; 193:849-880.

22. Cervera M, Oliver J, Faria R. Seismic evaluation of concrete dams via continuum damage models. Earthquake Engineering and Structural Dynamics 1995; 24:1225-1245.

23. Cervera M, Oliver J, Manzoli O. A rate-dependent isotropic damage model for the seismic evaluation of concrete dams. Earthquake Engineering and Structural Dynamics 1996; 25:987-1010.

24. Faria R, Oliver J, Cervera M. A strain-based plastic viscous-damage model for massive concrete structures. International Journal of Solids and Structures 1998; 35(14):1533-1558.

25. Faria R, Oliver J, Cervera M. Modeling material failure in concrete structures under cyclic actions. Journal of Structural Engineering (ASCE) 2004; 130:1997-2005.

26. Cervera M, Chiumenti M. Smeared crack approach: back to the original crack. International Journal for Numerical and Analytical Methods in Geomechanics 2006; 30:1173-1199.

27. Cervera M, Chiumenti M. Mesh objective tensile cracking via a local continuum damage model and a crack tracking technique. Computer Methods in Applied Mechanics and Engineering 2006; 196(1-3):304-320.

28. Simó JC, Ju JW. Strain- and stress-based continuum damage models-I. Formulation. International Journal of Solids and Structures 1987; 23:821-840.

29. de Borst R. Fracture in quasi-brittle materials: a review of continuum damage-based approaches. Engineering Fracture Mechanics 2002; 69:95-112.

30. Lemaitre J, Chaboche JL. Aspects phénoménologiques de la rupture par endommagement. Journal de Mecanique Appliquee 1978; 2:317-365.

31. Cervera M, Chiumenti M, Agelet de Saracibar C. COupled MEchanical and Thermal analysis. Data input manual, Version 5.0. Technical Report IT-308, 2002. Available from: www.cimne.upc.es.

32. GiD: The Personal Pre and Post-processor, 2006. Available from: www.gidhome.com. 